\title{
PERCUTANEOUS TRANSLUMINAL ANGIOPLASTY
}

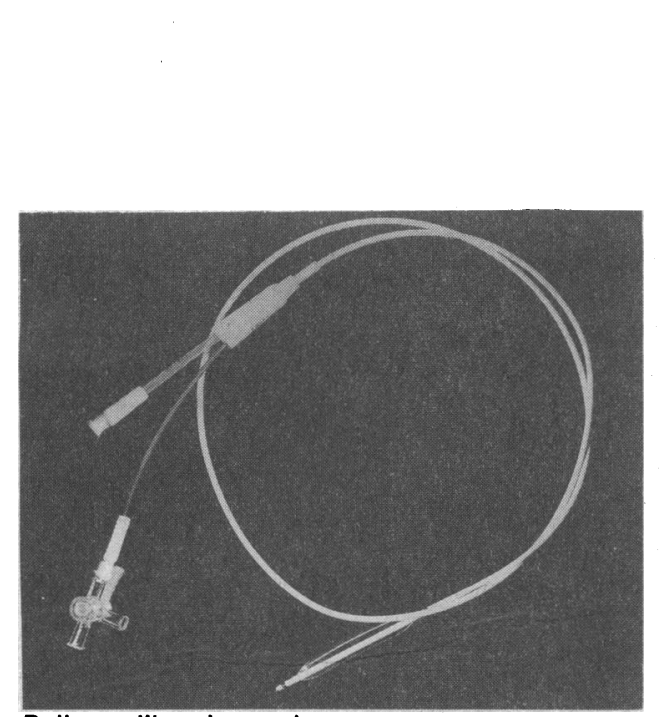

Balloon dilatation catheter.

\author{
M A Al-Kutoubi
}

Transluminal angioplasty was first described by Dotter and Judkins in 1964. They used coaxial catheters, but the technical limitations prevented its widespread acceptance until Gruntzig introduced the double lumen balloon catheter in 1976. This was both effective and easy to use, and established angioplasty as an acceptable technique for the treatment of vascular stenoses in most large arteries in the body. The simplicity and low associated morbidity have subtly altered the indications for intervention, but full clinical assessment of the patient and discussion with a vascular surgeon are essential before angioplasty is considered. There is no place for direct referral from a physician to a radiologist without consultation with a surgeon.

\section{Mechanism of dilatation}

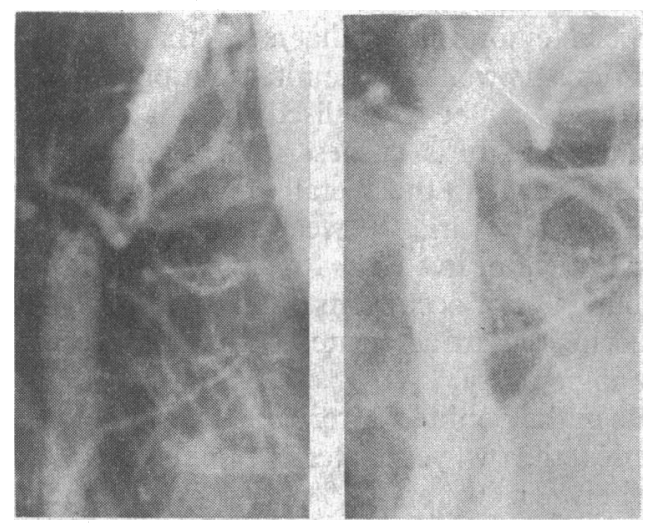

The stretching of the artery splits the intima, cracks the atheromatous plaques, and stretches - and sometimes ruptures - the media, causing local dissection and subintimal haemorrhage. Healing occurs by fibrosis and giant cell reaction with formation of neointima, resulting in a smooth lumen within a few weeks.

Right common iliac stenosis before (left) and after (right) balloon dilatation.

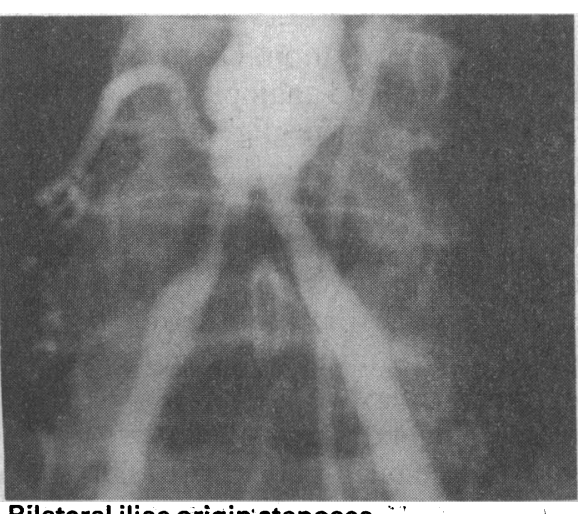

Bilateral iliac origin:stenoses.

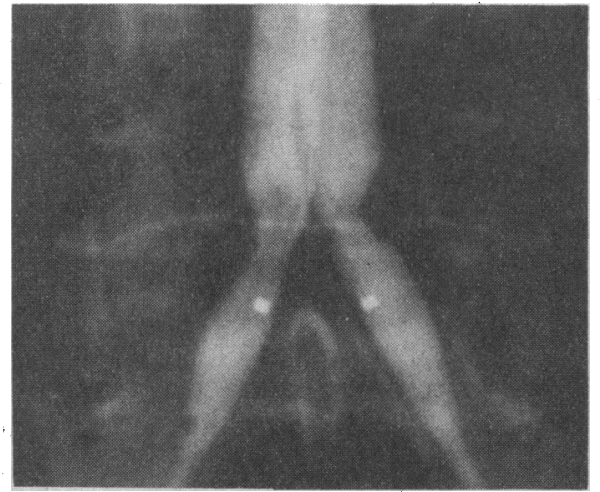

Bilateral iliac origin stenoses with "kissing" balloons in place.

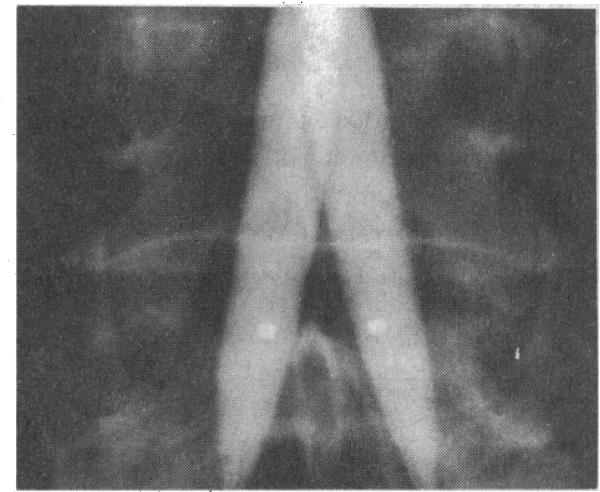

Bilateral iliac origin stenoses after plaque has been dilated. 


\section{Angioplasty of peripheral arteries}
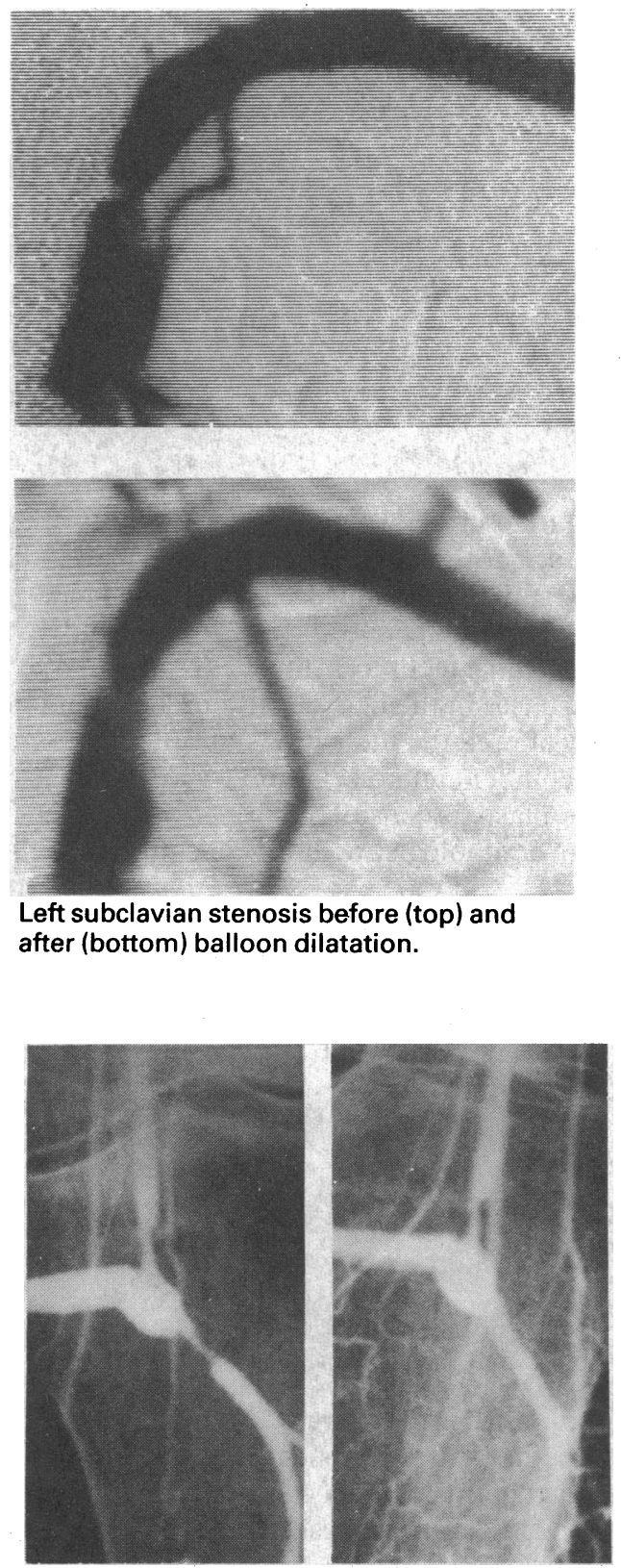

Stenosis at lower end of below knee femoropopliteal vein graft one year after operation before (left) and after (right) balloon dilatation.
Percutaneous transluminal angioplasty has been successfully used for the treatment of stenoses of the aorta and iliac, femoral, popliteal, and subclavian arteries; for treatment of stenosed grafts and arteriovenous dialysis shunts; as well as for stenoses of renal and mesenteric vessels and coronary arteries. Short occlusions of the femoropopliteal segment can be recanalised successfully, but short occlusions of the iliac artery respond less well.

\section{Antiplatelet drugs}

Antiplatelet drugs are probably important in improving the patency rate after angioplasty, and aspirin $75 \mathrm{mg}$ daily should be prescribed for six months after the procedure.

\section{Complications}

The associated morbidity varies from $5 \%$ to $15 \%$. Most complications are minor, such as a haematoma at the puncture site. Thrombosis of the vessel occurs in $2-3 \%$ of patients, as does distal embolisation. Perforation of the vessel and formation of a false aneurysm are rare. The procedure has to be abandoned because of technical difficulties in between $7 \%$ and $25 \%$ of cases.

\section{Results}

The immediate success of an angioplasty can be assessed during the examination by measuring the pressure proximal and distal to the stenosis and monitoring the radiological appearances.

The aortoiliac segment - The lesion that responds best to angioplasty is a symptomatic focal stenosis of the iliac artery. Special care must be taken when the stenosis affects the orifice of another vessel (such as the internal iliac artery) as the intimal split may result in a flap, which may occlude the orifice. At the aortic bifurcation particular care must be taken to prevent damage to the other side, and to minimise this risk the "kissing" balloon technique is used. Stenosis of the internal iliac arteries that are causing impotence have been dilated with reasonable results. Iliac occlusions are on the whole not suitable for angioplasty and are best treated by operation, but the initial success rate for angioplasty of iliac stenoses is about $96 \%$, and the five year patency has been reported to be as high as $90 \%$.

Superficial femoral artery-Angioplasty works best for a symptomatic localised stenosis of the superficial femoral artery that is less than $2 \mathrm{~cm}$ long, but most patients with such lesions do well with no intervention. Longer stenoses and occlusions (of $2-15 \mathrm{~cm}$ ) can be recanalised and dilated, however, with reasonable results. The initial success rate is significantly. better for stenoses than for occlusions, but the overall initial success rate is about $85 \%$. The long term patency also varies between stenoses and occlusions; it can be as high as $70 \%$ after five years for short segment stenoses, though it is only about $55 \%$ for occlusions. This is probably because longer stenoses and occlusions are associated with diffuse disease, making them less suitable for angioplasty.

Popliteal artery - The results in the popliteal arteries are similar to those in the superficial femoral arteries, the two year patency rate for the treatment of occlusions being between $60 \%$ and $70 \%$. Extreme care must be taken, however, with stenoses in the distal popliteal artery because damage may result in occlusion of one or more of the crural vessels.

\section{Angioplasty compared with operation}

Percutaneous transluminal angioplasty is:

- Useful in localised disease

- Causes minimal morbidity

- Can be repeated if necessary

Careful consideration and discussion with the vascular surgeon are an essential part of planning treatment
It is difficult to compare angioplasty with operation. On the whole, operation is reserved for more diffuse disease and angioplasty for localised stenoses. Mortality associated with angioplasty is negligible and morbidity is low, whereas mortality after operation ranges from $1 \%$ to $5 \%$ depending on the procedure. Important advantages of angioplasty are the short inpatient stay and the low cost. A successful femoral angioplasty costs about $£ 300$ and a successful bypass operation about $£ 1500$.

The advent of angioplasty has not, however, produced the expected savings, because more patients are now being investigated and treated. It complements rather than replaces open surgery.

Comparison of results is difficult not only because angioplasty is more suitable for patients with less severe disease but also because it fails in between $5 \%$ and $15 \%$ of patients because of technical reasons and these are usually excluded from analysis of results. Nevertheless, with favourable 


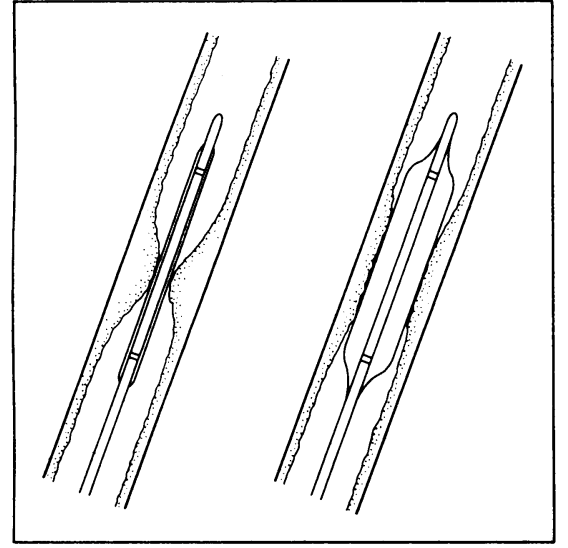

Balloon dilatation of an artery. lesions a five year success rate of $70 \%$ for femoropopliteal dilatations and $\mathbf{9 0 \%}$ for iliac dilatations (excluding technical failures) can be expected. These results compare favourably with the results for aortobifemoral and femoropopliteal grafting. It is important to recognise that the consequences of failure of angioplasty are not severe and recurrent stenoses can be redilated.

Angioplasty is now finding a role at the two extremes of the clinical range-for the patient with claudication caused by a discrete stenosis and for the patient with a critically ischaemic leg who represents a considerable operative risk. In addition, there is an increasing number of surgeons who like to perform an intraoperative iliac angioplasty before doing a femorodistal graft. Intraoperative injection of papaverine into the femoral artery (which results in relaxation of the arterioles and a reduction in femoral artery pressure if there is a more proximal stenosis) can identify those patients who require an additional procedure proximally, and angioplasty avoids retroperitoneal dissection of the iliac artery.

\section{Angioplasty combined with other techniques}

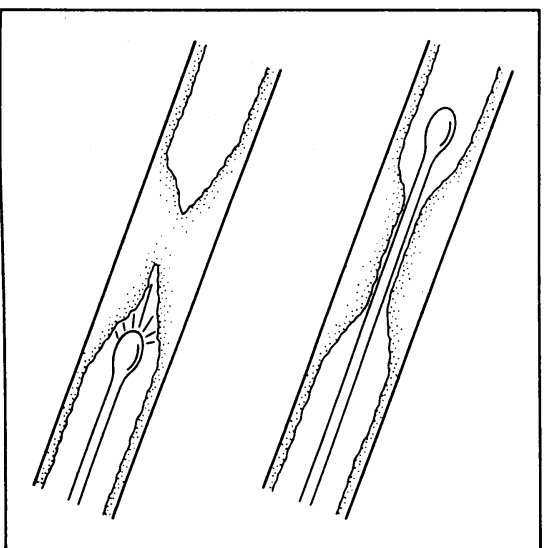

Laser used to create a channel. A balloon can then be used to dilate the track.

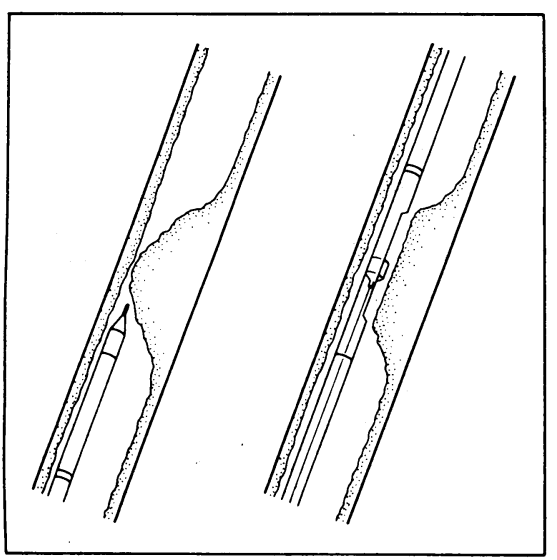

Eccentric plaque removed by atherectomy catheter.

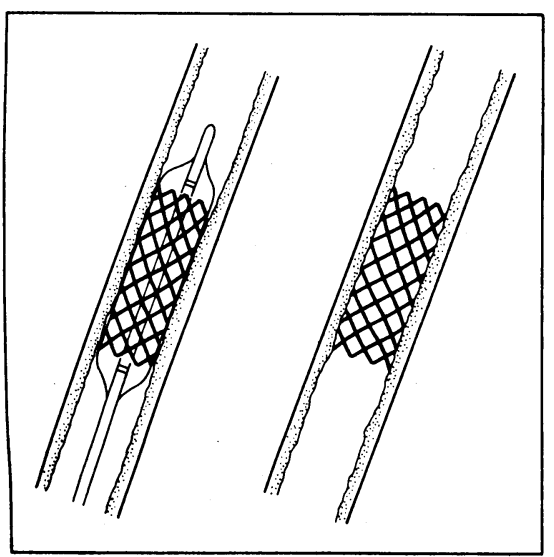

Stent inserted with a balloon catheter.

\section{Fibrinolysis}

Thrombolytic agents such as streptokinase can dissolve à recent thrombus in an artery and convert a long occlusion into a short stenosis that can be dilated by a balloon catheter. In one series the initial success rate was reported as $78 \%$ and the two year cumulative patency rate $81 \%$, but the use of thrombolytic agents is not without risk. Complications such as diffuse bleeding and allergic reactions may be fatal. A low dose local infusion of streptokinase ( $5000 \mathrm{U} / \mathrm{hour}$ ) reduces the systemic effect and can be useful before angioplasty in patients who are not suitable for operation. Urokinase and tissue plasminogen activator are more specific, and are not associated with allergic reactions, but they are 20 times more expensive than streptokinase. Furthermore, the current data do not show that they have any particular practical advantage.

\section{Laser assisted angioplasty}

The "hot tip" laser converts the laser energy into heat at the metal tip of the laser fibre, which burns atheroma inside the artery. Other systems use more direct laser energy and produce less damage to the vessel wall. Laser treatment was hailed as an important advance in the ablation of atheroma, but the long term results are worse than for conventional balloon procedures. The current use of lasers is limited to long occlusions in which the laser probe is used to create a channel through which balloon catheters can be advanced and balloon angioplasty done.

\section{Atheroma cutters}

High speed revolving cutters have been developed that core out atheromatous arteries; the Simpson atherectomy device was introduced in 1985 and has a side window that is positioned against the atheromatous plaque, which is then removed by the high speed cutter. The atheromatous material is stored in a special chamber in the catheter and is later removed for histopathological examination. This is ideal for localised eccentric plaques and stenoses, and a two year patency rate of $83-90 \%$ has been reported in localised iliac and femoral lesions. Other systems incorporate a rotary cutting edge at the tip of the catheter which cores out a channel through an occluded artery to facilitate the advancement of a balloon catheter.

\section{Vascular stents}

Vascular stents were developed to maintain a patent arterial lumen, but the metal mesh does not prevent reaccumulation of atheroma in the lumen of the artery and intimal hyperplasia seems to form more rapidly at the ends of the stents. They are mainly used to treat recurrent stenoses or dissections, particularly in the iliac arteries, for which one year patency rates of up to $86 \%$ have been reported. The stents are not as effective in the femoral arteries, in which $57 \%$ patency after one year has been reported.

We acknowledge with thanks the assistance of the audiovisual department, St Mary's Hospital, London, in the preparation of the illustrations. 